\title{
Editorial
}

\section{Tuberculosis: The Past, the Present and the Future}

\author{
Coenraad F.N. Koegelenberg ${ }^{\mathrm{a}}$ Otto D. Schoch ${ }^{\mathrm{b}, \mathrm{c}}$ Christoph Lange $^{\mathrm{d}, \mathrm{e}, \mathrm{f}, \mathrm{g}}$ \\ a Division of Pulmonology, Department of Medicine, Stellenbosch University and Tygerberg Hospital, Cape Town, \\ South Africa; ${ }^{b}$ Department of Pneumology and Sleep Medicine, Kantonsspital St. Gallen and University of Zurich, \\ Zurich, Switzerland; 'Tuberculosis Competence Center, Swiss Lung League, Berne, Switzerland; d Division of

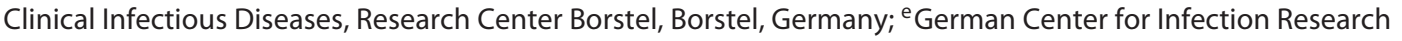

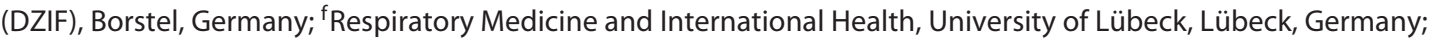 \\ gGlobal TB Program, Baylor College of Medicine, Houston, TX, USA
}

The current coronavirus disease 2019 (COVID-19) pandemic caused by the severe acute respiratory syndrome coronavirus-2 (SARS-CoV-2) has not only highlighted the vulnerability of humans to infectious agents and the massive health and economic impact contagious diseases can have on life on planet earth, but also put the global disease burden from tuberculosis into greater perspective [1]. COVID-19 started as a small outbreak in Wuhan, China, soon spread to all continents, infecting more than 150 million people and causing more than 3 million deaths, thereby surpassing the estimated number of annual tuberculosis deaths in 2020, and for the first time in many years, taking the world-wide lead as a cause of death by a single infectious disease [2]. COVID-19 has precipitated major scientific advances, but unfortunately also had a negative impact on the diagnosis and management of other diseases [3].

Even early in the COVID-19 pandemic, the Stop TB partnership and others warned of the potential devastating impact the pandemic could have on tuberculosis control [4-6]. The combination of lockdown measures and the shear excess disease burden placed on poorly resourced health care systems in low- and middle-income countries led many to believe that tuberculosis control, based on various modelling analyses, will be severely affected in the regions who could ill afford it, and that the "collateral damage" would be felt for many years to come $[5,7]$. Other concerns included that the comorbidities often found in patients with tuberculosis and the disease itself could lead to severe COVID-19 [5]. Their fears, as far as tuberculosis case detection is concerned, were unfortunately realised in 2020, but a strong association between active pulmonary tuberculosis and severe COVID-19 was not observed in high-incidence settings [3, 8]. Comorbidities often seen in tuberculosis, notably human immunodeficiency virus (HIV) and especially diabetes mellitus, were shown to be associated with severe COVID-19 [8, 9].

According to the latest estimations, approximately 25\% of the world's population are infected with Mycobacterium tuberculosis [10]. The World Health Organization (WHO) estimated that the global incidence of tuberculosis peaked around 2003 and appeared to be declining slowly until 2019 , and that 1.6 million people died of tuberculosis in

From the Thematic Review Series: "Tuberculosis." Series Editors: Coenraad F. Koegelenberg, Christoph Lange, Otto D. Schoch.
Correspondence to:

Coenraad F.N. Koegelenberg, coeniefn@sun.ac.za 
2019 [3]. The COVID-19 pandemic has unfortunately reversed the steady downward trend, as an alarmingly 50\% drop in tuberculosis case detection in many parts of the world was reported in 2020, which was highly unlikely a true reflection of the state of affairs but rather a result of major delays in the diagnosis as health-care systems were overrun with cases of COVID-19 [3]. It is estimated that this indirect impact of COVID-19 resulted in 400,000 additional tuberculosis deaths during 2020 [3].

The greatest challenges in the fight against the tuberculosis epidemic prior to COVID-19 remained poor socioeconomic circumstances (including access to health care), HIV infection and M. tuberculosis drug resistance [11]. These factors have led to a very unequal incidence of tuberculosis around the globe, with the greatest disease burden in India and China, the highest incidence rates seen in sub-Saharan Africa [11] and the highest rates of drug-resistant tuberculosis in countries of the former Soviet Union [3]. The inequality is highlighted by the fact that 8 countries accounted for two-thirds of the global total: India (26\%), Indonesia (9\%), China (8\%), the Philippines (6\%), Pakistan (6\%), Nigeria (4\%), Bangladesh (4\%) and South Africa (4\%) [3]. The WHO End TB Strategy was a resolution that set out several goals in 2014, but the WHO has already acknowledged that the 2020 goals, which included a $20 \%$ reduction in incidence between 2015 and 2020, were not met [3]. Tuberculosis will, therefore, remain a global epidemic for many decades to come.

The journal Respiration was first published in 1944 under the banner Schweizerische Zeitschrift für Tuberkulose (Swiss Journal for Tuberculosis, 1944-1955) and later Schweizerische Zeitschrift für Tuberkulose und Pneumologie (Swiss Journal for Tuberculosis and Pneumology, 1956-1967), highlighting the important role that tuberculosis played in Switzerland and the rest of Europe at the time. With socioeconomic upliftment, improvements in health care and the advent of anti-tuberculosis agents, tuberculosis went from a common to a rare disease in much of the western world, and interest in novel diagnostic techniques and treatment modalities soon waned, as almost complete eradication seemed inevitable [12]. Sadly, this was not the case in much of the developing world.

In the next issues of Respiration, we shall return to the roots of the journal and highlight the topic of tuberculosis in a thematic review series, from the Ghon focus and its relevance today all the way to novel developments into the diagnosis and management of the disease [13]. Furthermore, we will put the very under-recognised entity of post-tuberculosis lung disease (PTLD) in the spotlight [14].
In the first review in the series, Peter Donald et al. [13] critically review the work of Anton Ghon and how it aided our understanding of the disease. His ground-breaking publication, "The Primary Lung Focus of Tuberculosis in Children," is still often referenced but not infrequently misquoted [15]. The work of Ghon and his contemporaries has aided us in understanding primary pulmonary tuberculosis, particularly its primary, often subpleural foci and the subsequent lympho-haematogenous spread. Many of the theories proposed by Ghon solely based on pathological observations were ultimately proved to be correct using modern molecular and imaging techniques [13].

A crucial aspect of achieving tuberculosis control is the detection and diagnosis of infectious and active cases, thereby interrupting the transmission chain for M. tuberculosis [16]. Widespread use of screening tools for the key symptoms of tuberculosis and novel screening technology, aided with artificial intelligence, as well as novel biological markers have recently been developed and are now ready for wider study and implementation in highprevalence countries for tuberculosis [17-19]. The review of these diagnostic aspects by Grant Theron et al. [under review] will shed light on future developments.

Defining treatment outcomes in tuberculosis remains a controversial topic. The review by Gunar Günther et al. [20] puts different definitions of cure from tuberculosis, including the one currently proposed by the WHO, into clinical perspective, pointing out the many challenges of these definitions. These definitions mostly rely on sputum results, which become problematic if no sputum is produced. The authors review alternative definitions, including continuation of treatment as a proxy of failure. In the future, individualisation of treatment and its duration will depend on pathogen- or host-specific biomarkers, which are currently being investigated $[20,21]$.

After just over half a century of inertia in the field of antituberculous drug development, the novel agent bedaquiline (initially known as TMC207) was described in 2005 and found to have in vitro activity against the ATP synthase of M. tuberculosis [22]. It took almost another decade for human trials to confirm its utility in drug-resistant tuberculosis [23]. Other novel agents have been introduced since, and other well-known antibiotics, for example, linezolid or clofazimine, have been "refurbished" for use in various regimens with the aim of shortening treatment duration and overcoming resistance [24]. In a further review in this series, Martin Boeree et al. [under review] will provide a balanced overview of both 
the novel agents as well as the inclusion of existing antibiotics into current anti-tuberculosis treatment regimens.

Bacteriophages are viruses that are exclusively dependent on the metabolism of bacteria, which they eventually kill, and are the most ubiquitous organisms on the planet [25]. Thousands of mycobacteriophages have been isolated that target and kill mycobacteria independent of antibiotic resistance, although only a small number of these are active against M. tuberculosis. Andreas Diacon et al. [under review] will review our current knowledge about and review the chances and obstacles for mycobacteriophages to become available for tuberculosis treatment in future.

Almost 60 million people have survived tuberculosis since the turn of the century and a significant number of them still have some degree of impairment due to PTLD [14]. PTLD encompasses a wide spectrum of afflictions ranging from large and small airways disease (bronchiectasis and obstructive lung disease) to parenchyma involvement, pulmonary vascular disease, pleural disease and co-infections [14]. PTLD is also associated with a shortened life expectancy and increased risk of recurrent tuberculosis. The more severe the restriction in lung function, the higher the likelihood of a negative treatment outcome [26]. Very little real evidence exists on how to optimally manage these patients, and most interventions are based on other similar conditions (e.g., non-cystic fibrosis bronchiectasis). In the final review, Brian Allwood et al. [14] will highlight the spectrum of disease, the lack of evidence and the great need for advocacy for this neglected entity.

We trust the readers of Respiration will find the selection of articles balanced and will enjoy the journey through the thematic review series "Tuberculosis."

\section{Conflict of Interest Statement}

The authors have no conflicts of interest to declare.

\section{Funding Sources}

No funding was received.

\section{Author Contributions}

C.F.N.K. wrote the first draft, which was critically reviewed and edited by all authors.

\section{References}

1 Sanche S, Lin YT, Xu C, Romero-Severson E, Hengartner N, Ke R. High Contagiousness and Rapid Spread of Severe Acute Respiratory Syndrome Coronavirus 2. Emerg Infect Dis. $2020 \mathrm{Jul} ; 26(7): 1470-7$.

2 Johns Hopkins University. Corona Virus Resource Center. 2020. Available from: https:// coronavirus.jhu.edu/map.html.

3 WHO. Global Tuberculosis Report 2020. 2020. Available from: https://www.who.int/ teams/global-tuberculosis-programme/tbreports/global-tuberculosis-report-2020.

4 Stop TB partnership. The Potential Impact of the Covid-19 Response on Tuberculosis in High-Burden Countries: a Modelling Analysis. Developed by Stop TB Partnership in Collaboration with Imperial College, Avenir Health, Johns Hopkins University and USAID. 2020;(May):1-7. Available from: http:// www.stoptb.org/assets/documents/news/ ModelingReport_1May2020_FINAL.pdf.

5 Togun T, Kampmann B, Stoker NG, Lipman M. Anticipating the impact of the COVID-19 pandemic on TB patients and TB control programmes. Ann Clin Microbiol Antimicrob. 2020 May;19(1):21.

6 McQuaid CF, McCreesh N, Read JM, Sumner T, Houben RM, White RG, et al.; CMMID COVID-19 Working Group. The potential impact of COVID-19-related disruption on tuberculosis burden. Eur Respir J. 2020 Aug; 56(2):2001718.

7 Udwadia ZF, Vora A, Tripathi AR, Malu KN, Lange C, Sara Raju R. COVID-19-Tuberculosis interactions: when dark forces collide. Indian J Tuberc. 2020 Dec;67(4S):S155-62.

8 Parker A, Koegelenberg CF, Moolla MS, Louw EH, Mowlana A, Nortjé A, et al. High HIV prevalence in an early cohort of hospital admissions with COVID-19 in Cape Town, South Africa. S Afr Med J. 2020 Aug; 110(10):982-7.

9 Boulle A, Davies MA, Hussey H, Ismail M, Morden E, Vundle Z, et al. Risk factors for COVID-19 death in a population cohort study from the Western Cape Province, South Africa. Clin Infect Dis. 2020 Aug;29:ciaa1198.

10 Houben RM, Dodd PJ. The Global Burden of Latent Tuberculosis Infection: A Re-estimation Using Mathematical Modelling. PLoS Med. 2016 Oct;13(10):e1002152.

11 Corbett EL, Marston B, Churchyard GJ, De Cock KM. Tuberculosis in sub-Saharan Africa: opportunities, challenges, and change in the era of antiretroviral treatment. Lancet. 2006 Mar;367(9514):926-37.

12 Diacon $\mathrm{AH}$, von Groote-Bidlingmaier $\mathrm{F}$, Donald PR. From magic mountain to table mountain. Swiss Med Wkly. 2012 Aug;142: w13665.

13 Donald PR, Diacon AH, Thee S. Anton Ghon and His Colleagues and Their Studies of the Primary Focus and Complex of Tuberculosis Infection and Their Relevance for the TwentyFirst Century. Respiration. 2021;100:557-567.

14 Allwood BW, Byrne A, Meghii J, Rachow A, van der Zalm MM, Schoch OD. Post-Tuberculosis Lung Disease: Clinical Review of an Under-Recognised Global Challenge. Respiration. 2021, DOI: 10.1159/000512531.

15 Ghon A. Der primäre Lungenherd bei der Tuberkulose der Kinder. Urban \& Schwarzenberg; 1912.

16 Tuberculosis: a much-needed focus on transmission. Lancet Respir Med. 2019 Jun;7(6): 465.

17 Mor Z, Leventhal A, Diacon AH, Finger R, Schoch OD. Tuberculosis screening in immigrants from high-prevalence countries: interview first or chest radiograph first? A pro/con debate. Respirology. 2013 Apr;18(3):432-8.

18 Qin ZZ, Sander MS, Rai B, Titahong CN, Sudrungrot S, Laah SN, et al. Using artificial intelligence to read chest radiographs for tuberculosis detection: A multi-site evaluation of the diagnostic accuracy of three deep learning systems. Sci Rep. 2019 Oct;9(1):15000. 
19 Broger T, Nicol MP, Sigal GB, Gotuzzo E, Zimmer AJ, Surtie S, et al. Diagnostic accuracy of 3 urine lipoarabinomannan tuberculosis assays in HIV-negative outpatients. J Clin Invest. 2020 Nov;130(11):5756-64.

20 Günther G, Heyckendorf J, Zellweger JP, Reimann M, Claassens M, Chesov D, et al. Defining outcomes of tuberculosis treatment from the past to the future. Respiration. 2021, DOI: $10.1159 / 000516392$.

21 Heyckendorf J, Marwitz S, Reimann M, Avsar K, DiNardo A, Günther G, et al. Prediction of anti-tuberculosis treatment duration based on a 22-gene transcriptomic model. Eur Respir J. 2021 Feb;2003492:2003492.
22 Andries K, Verhasselt P, Guillemont J, Göhlmann HW, Neefs JM, Winkler H, et al. A diarylquinoline drug active on the ATP synthase of Mycobacterium tuberculosis. Science. 2005;307(5707):223-7.

23 Diacon AH, Pym A, Grobusch MP, de los Rios JM, Gotuzzo E, Vasilyeva I, et al.; TMC207C208 Study Group. Multidrug-resistant tuberculosis and culture conversion with bedaquiline. N Engl J Med. 2014 Aug;371(8):72332.
24 Mirzayev F, Viney K, Linh NN, Gonzalez-Angulo L, Gegia M, Jaramillo E, et al. World Health Organization recommendations on the treatment of drug-resistant tuberculosis, 2020 update. Eur Respir J. 2020 Nov;2003300.

25 D'Accolti M, Soffritti I, Mazzacane S, Caselli E. Bacteriophages as a Potential 360-Degree Pathogen Control Strategy. Microorganisms. 2021 Jan;9(2):261.

26 Chesov D, Butov D, Reimann M, Heyckendorf J, Myasoedov V, Butov T, et al. Impact of lung function on treatment outcome in patients with TB. Int $\mathrm{J}$ Tuberc Lung Dis. 2021;25(4):277-84 